\title{
Long-term effects of the Dutch Schoolgruiten Project - promoting fruit and vegetable consumption among primary-school children
}

\author{
Nannah I Tak ${ }^{1, *}$, Saskia J te Velde ${ }^{1}$ and Johannes Brug ${ }^{1,2}$ \\ 'EMGO-institute, Department of Epidemiology \& Biostatistics, VU University Medical Centre, Van der \\ Boechorststraat 7, 1081 BT Amsterdam, The Netherlands: ${ }^{2}$ Department of Public Health, Erasmus MC, \\ University Medical Centre Rotterdam, Rotterdam, The Netherlands
}

Submitted 4 October 2007: Accepted 18 August 2008: First published online 22 October 2008

\begin{abstract}
Objectives: To evaluate the long-term effects of the Schoolgruiten Project, a Dutch primary school-based intervention providing free fruit and vegetables (F\&V). In addition, we assessed whether children's appreciation of the project mediated these intervention effects.

Design and methods: Participating schoolchildren (mean age 9.9 years at baseline) and their parents completed parallel questionnaires at baseline, at 1-year and at 2-year follow-up, including questions on usual F\&V intake of the child, potential behavioural determinants, their appreciation of the project and general demographics. Primary outcomes were usual F\&V intakes as assessed by parent and child self-reported food frequency measures. Secondary outcome measures were taste preference, knowledge of daily recommendations, availability and accessibility for fruit intake. Multilevel linear regression analyses were used to assess differences at second follow-up adjusted for baseline values between control and intervention groups.

Subjects: Reports were available for 346 intervention children (148 parents) and 425 control children (287 parents).

Results: Both child and parent reports indicated that the intervention group had a significantly higher fruit intake at 2-year follow-up (difference, servings/d: $0 \cdot 15$; $95 \%$ CI $0.004,0.286$ for child reports; $0 \cdot 19$; $95 \%$ CI $0.030,0.340$ for parent reports). No significant effects on vegetable intake were observed. Significant positive intervention effects were also found for knowledge of fruit recommendations among boys. Some evidence was found for partial mediation analyses of the effects on fruit intake.

Conclusion: The present study indicates that the Schoolgruiten scheme was effective in increasing children's fruit intake and that appreciation of the project partially mediated this effect.
\end{abstract}

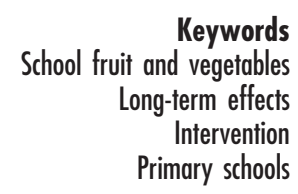

Since most children in Western countries, including The Netherlands, do not comply with recommendations for fruit and vegetable $(\mathrm{F} \& \mathrm{~V})$ intake, several interventions promoting F\&V consumption have been implemented.

One of the Dutch interventions is the Schoolgruiten Project. Schoolgruiten is a Dutch acronym for 'school fruits and vegetables' and is the largest-scale free F\&V scheme for Dutch primary-school children. The main strategy of this project is to improve accessibility of F\&V at school by providing a serving of fruit or vegetable for free to all children twice weekly. The Schoolgruiten Project is meant to grow into a nationwide campaign for primary-school children, but started with a pilot phase in which the intervention was tested in a controlled design, to inform further improvement of the intervention or justify further implementation. Effect evaluation at 1-year follow-up showed mixed results, i.e. a significantly higher vegetable intake (difference of $20.7 \mathrm{~g} / \mathrm{d}$ ) for children of non-Western ethnicity and a significantly higher fruit intake for children of Dutch ethnicity (difference of 0.23 pieces/d), both based on child reports. No significant effects on intake were observed based on parent reports. Significant positive intervention effects were also found for perceived accessibility among children of non-Western ethnicity, and for parent-reported taste preference of their child among children of non-Western ethnicity and boys of Dutch ethnicity $^{(1)}$.

Nutrition behavioural change interventions such as Schoolgruiten can only have an impact on health if effects are maintained or improved over time and longer-term evaluations are therefore warranted. Furthermore, to inform future intervention schemes, it is of additional 
importance to explore possible mediators of intervention effects, i.e. to identify effect-enhancing characteristics of the intervention. Previous research indicated that children's appreciation of the project was associated with intervention effects ${ }^{(2)}$.

The current study assessed the 2-year follow-up effects of the Schoolgruiten F\&V scheme on intake and potential behavioural determinants (knowledge of recommended intake levels, taste preferences, availability and accessibility of F\&V at home) $)^{(3-6)}$, and explored whether children's appreciation of the project mediated these intervention effects. We hypothesized that the children of the intervention group would have higher $\mathrm{F} \& \mathrm{~V}$ intakes and more positive scores on the main determinants of $F \& V$ than the children of the control group. Furthermore, we hypothesized that children in the intervention group would report higher appreciation for $\mathrm{F} \& \mathrm{~V}$-promoting projects than children in the control group and, additionally, that children's appreciation of the project would mediate the intervention effects.

\section{Methods}

\section{The Schoolgruiten Project}

Since earlier studies and reviews indicated that taste preferences, availability and accessibility are important determinants of F\&V consumption among children ${ }^{(4,6)}$ and because intakes should be promoted through changes in such presumed mediators ${ }^{(7)}$, the main strategies within the Schoolgruiten Project targeted these factors.

The main component of the Schoolgruiten Project was an F\&V scheme improving the availability, accessibility and exposure to $\mathrm{F} \& \mathrm{~V}$ at school. The children in the intervention group received a piece of fruit or readyto-eat vegetables (cherry tomatoes, baby carrots) for free twice a week during a fruit break. The aim of the Schoolgruiten Project was that all children eat the piece of fruit or vegetable together in their own classroom. Apart from increasing availability and accessibility, this F\&V scheme was also supposed to increase the children's exposure to $\mathrm{F} \& \mathrm{~V}$. Repeated exposure is an important determinant of taste preferences ${ }^{(8)}$.

Additionally, a school curriculum developed and carefully pre-tested by the Netherlands Nutrition Centre Foundation, aimed at increasing knowledge and skills related to $\mathrm{F} \& \mathrm{~V}$ consumption, was offered to the intervention schools. The schools were not obliged to use this curriculum, but they were strongly encouraged to do so.

\section{Recruitment of the schools and study sample}

The Schoolgruiten Project was implemented in seven cities in The Netherlands. These cities were indicated by the Dutch Ministry of Public Health, Welfare and Sport. Because of time and financial constraints only two of these cities were included in the evaluation study. These were one big city (The Hague) in the western part of the
Netherlands and a smaller town (Almelo) in the eastern part. Therefore these cities represent different parts of The Netherlands and have very distinct characteristics, The Hague being a major city while Almelo has more rural characteristics.

The design of the evaluation study was quasi experimental, with a pre- and post-test, and an intervention and control group. Since the intervention cities were decided upon by the authorities, no randomisation was possible. The Schoolgruiten research group selected three control cities: Zoetermeer and Leidschendam close to The Hague, and Hengelo, which is close to Almelo. In the western region it was nessecary to select two control cities in order to recruit enough children.

All fourth grades (9-10 years) from primary schools in the five cities were eligible for participation at baseline, and schools were randomly approached by telephone and invited to participate in this survey. Recruitment ended when fifty-five primary schools had agreed to participate in the survey, of which thirty-one were intervention schools and twenty-four were control schools, ensuring a sample of at least 600 children of the 4th grade in the intervention as well as in the control group.

For one city (Hengelo) records were kept to assess school willingness to participate. Sixteen schools were invited to participate in that city of which half agreed immediately, four refused and another four schools had to consult their external school board before confirming participation. Only the eight schools that agreed immediately were included in the study. Similar procedures and rates of agreement were found in the other cities.

The baseline survey was conducted prior to the start of the intervention. First follow-up among the same children was exactly one year later and second follow-up was conducted exactly two years later. The baseline survey was conducted in The Hague, Zoetermeer and Leidschendam (western region) in the spring of 2003 and in Almelo and Hengelo (eastern region) in the autumn of 2003.

For this study both children as well as their parents completed questionnaires about the child's intake, potential determinants, general demographics and their general opinion about the Schoolgruiten Project.

\section{Procedure}

Children completed the questionnaire within one school hour guided by their own teacher in their classroom, based on a written administration protocol provided by the research staff. The children brought home a parent questionnaire to be completed preferably by the parent usually taking care of the child's meals. The children got a small gift when they returned the completed parent questionnaire.

All fourth graders who were present on the day and hour of administration completed the questionnaires at baseline (1328 children, response rate of 100\%; 1070 parents, response rate of $81 \%$ ). 
Five schools were not willing to participate any longer at first follow-up, resulting in fewer children ( $n$ 1140, response rate of $86 \%$ ) and parents ( $n$ 931, response rate of $70 \%$ ). Six schools were no longer willing to participate at second follow-up, again reducing the number of children ( $n$ 792, response rate of $60 \%$ ) and parents ( $n$ 431, response rate of $32 \%$ ).

Finally, a total of 346 intervention and 425 control children had valid self-reported data for all three measurements and were included for analyses. Children with valid self-reported data on fruit and/or vegetable intake at baseline but not at first or at second follow-up were considered as dropouts. Dropout was due to the loss of five schools at first follow-up ( $n$ 112) and the loss of six schools at second follow-up ( $n$ 91) and because children moved to other places or schools, did not graduate to the next grade, were sick on the day of administration at first ( $n$ 194) or second follow-up ( $n$ 115), or had missing F\&V reports at first ( $n$ 23) or second follow-up ( $n$ 22).

Regarding parents, data were available for 148 intervention parents and 287 control parents. Dropout was due to the loss of five schools at first follow-up ( $n$ 105) and the loss of six schools at second follow-up ( $n$ 90), parents who moved, had a child who did not graduate to the next grade or who was sick at the day of administration, and parents who refused to complete the questionnaire at first ( $n$ 218) or second follow-up ( $n$ 218), or had missing F\&V reports at first ( $n$ 2) or second follow-up ( $n$ 2).

Responses were treated anonymously and confidentiality was ensured. The Schoolgruiten study was approved by the Netherlands Organization for Health Research and Development (ZonMw) Program for Prevention and the World Cancer Research Fund.

\section{Questionnaires}

Separate questionnaires for children and parents were developed, both based on and thus similar to the validated questionnaire of the Pro Children Study ${ }^{(9,10)}$. By parallel questions in the two questionnaires, the usual intake of F\&V among the children was assessed with the Pro Children food frequency questions. Potential determinants of $F \& V$, such as taste preference, knowledge of recommendations, accessibility and availability, were assessed with questions similar to those used in the Pro Children Study ${ }^{(5)}$.

The parent questionnaire also included questions on the parent's country of birth, level of education, age, child's age and number of siblings. Information on the country of birth of parents was used to make distinctions between children of Dutch, non-Western and non-Dutch Western ethnicity ${ }^{(11)}$. Educational level was divided into three categories based on the highest educational level of one of the parents (primary school or pre-vocational training $=$ low; high school or medium-level vocational training = medium; high-level vocational training, college or university training $=$ high).
A more detailed description of the questions and answer alternatives of both questionnaires has been published previously ${ }^{(12)}$.

\section{Appreciation of the project}

To assess children's appreciation of the project or of an $\mathrm{F} \& \mathrm{~V}$-promoting project in general, one open question was included in the child questionnaire in both the intervention and the control group. At baseline the question was 'Do you think it is a good idea for readyto-eat F\&V to be provided at your school?' In the control group the question was the same at both follow-ups, but in the intervention group the question was adapted at both follow-ups to "What do you think about the project Schoolgruiten?' The answers were re-coded to a threepoint scale: negative $(-1)$, neutral $(0)$ and positive $(+1)$.

\section{Statistical metbods}

Since in the present project both child- and parent-report data were available and it is not clear which data are most valid and sensitive for evaluation of school-based interventions $^{(12,13)}$, all analyses were performed on both data sets.

We used a complete cases design and therefore included only children and parents who had valid fruit intake or vegetable intake on all three measurements. Quitting the study was not a conscious decision of the child, but a result of circumstances not influenced by the child, e.g. moving to another school or town, not graduating to the next grade or a decision of the school board to quit the study.

Selective dropout bias and selective parent participation bias were assessed by logistic regression analyses with gender, ethnicity, parent educational level, region of residence of the children (categorical variables) and intake of fruit or vegetables at baseline (continuous variables) as independent variables and dropout ( 1 = yes, $0=$ no) as the dependent variable.

Means, standard deviations and percentages were calculated to describe the key variables.

To describe unadjusted outcomes, paired-sample $t$ tests, $t$ tests for independent samples, paired Wilcoxon tests and $\chi^{2}$ tests were used. To assess the adjusted effect of the intervention regarding the primary outcomes, multilevel linear regression analyses were performed to compare fruit or vegetable intakes at second follow-up (dependent variable) between intervention and control groups (dichotomous independent variable, scored as 1 and 0 , respectively). A multilevel analysis was used to take into account that effects may cluster within schools/classes. Analyses were further adjusted for children's age, gender, parents' education level, region of residence and baseline intake levels. The estimated regression coefficient reflects the adjusted difference in fruit/vegetable consumption at second follow-up between the intervention and control group. The residuals of the regression analyses were checked for normality and were considered as acceptable. 
Effect modification by gender, ethnicity, educational level and region of residence was assessed by including gender $\times$ group, ethnicity $\times$ group, educational level $\times$ group and region of residence $\times$ group interaction terms in the model. When these terms approached significance $(P<0 \cdot 05)$, analyses were stratified.

Intervention effects on the main determinants of $\mathrm{F} \& \mathrm{~V}$ intake, which were all categorical variables, were assessed by means of multilevel multinomial regression analyses, as suggested by Twisk and Proper ${ }^{(14)}$. Therefore, newly categorical variables were constructed, with three categories which were defined by the scores on baseline and second follow-up. The categories were: the 'stable high and increased' group (=the reference group (0)), the 'stable low' group (1) and the 'decreased' (2) group. Again, group (intervention $=1$, control $=0$ ) was the independent variable and the analyses were adjusted for children's age, gender, education level of the parents and region of residence. The estimated odds ratios reflect the odds for the intervention group of being in the specific category compared with being in the reference category

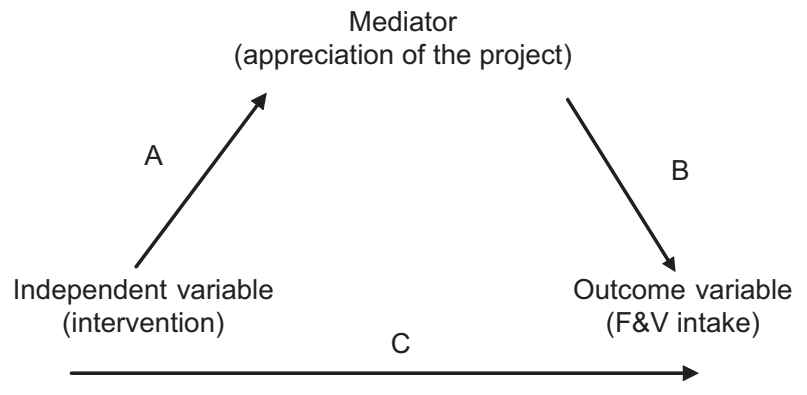

Fig. 1 Mediation model used to evaluate the long-term effects of the Schoolgruiten Project, a Dutch primary school-based intervention providing free fruit and vegetables (F\&V) (=stable high/increased). When cells for the multinomial logistic regression analyses include a small number, no reliable odds ratios can be estimated. Five per cent of the total sample or less was considered as a small number and in that case three categories were merged into two categories to solve this problem.

Regression analyses were further used to identify mediation by the appreciation of the project. Several authors describe criteria that must be met for a variable to be considered a mediator ${ }^{(15-18)}$. In the present study this implies that (see Fig. 1): (i) the independent variable (intervention) must be independently associated with the presumed mediator (appreciation of the project; path A); (ii) the presumed mediator must be independently associated with the dependent variable (F\&V intake; path B); (iii) the intervention must be associated with $\mathrm{F} \& \mathrm{~V}$ intake (path C); and (iv) the association between the intervention and F\&V intake must decrease substantially when adjustment is made for appreciation of the project. To determine the associations between the potential mediator and F\&V intake, mediator models, controlled for intervention, were applied.

The descriptive and unadjusted data analyses were performed using the SPSS statistical software package version 14.0 (SPSS Inc., Chicago, IL, USA, 1999). The multilevel analyses were conducted using MLwiN software version $2 \cdot 01^{(19)}$. The significance level was set at $P<0 \cdot 05$.

\section{Results}

\section{Dropout and non-participating parents}

Owing to the loss of eleven schools (eight intervention schools and three control schools), selective dropout was found for children in the intervention group (child

Table 1 Characteristics of the study population at baseline (child data): participants in the Schoolgruiten Project, a Dutch primary school-based intervention providing free fruit and vegetables

\begin{tabular}{|c|c|c|c|c|c|c|c|}
\hline \multirow[b]{2}{*}{ Characteristic } & \multicolumn{3}{|c|}{ Intervention group } & \multicolumn{3}{|c|}{ Control group } & \multirow[b]{2}{*}{$P$-value* } \\
\hline & $n$ & Mean or \% & SD & $n$ & Mean or $\%$ & SD & \\
\hline Age of the children (years) & 346 & $10 \cdot 1$ & 0.6 & 425 & $9 \cdot 9$ & 0.5 & 0.162 \\
\hline Age of the parents (years) & 249 & $38 \cdot 1$ & $5 \cdot 2$ & 361 & $40 \cdot 1$ & $4 \cdot 9$ & 0.472 \\
\hline \multicolumn{8}{|l|}{ Gender (\%) } \\
\hline Boys & 148 & $42 \cdot 8$ & & 198 & $46 \cdot 6$ & & $0 \cdot 290$ \\
\hline Girls & 198 & $57 \cdot 2$ & & 227 & $53 \cdot 4$ & & \\
\hline \multicolumn{8}{|l|}{ Ethnicity (\%) } \\
\hline Native Dutch children & 135 & $39 \cdot 0$ & & 301 & $70 \cdot 8$ & & $<0.001$ \\
\hline Children of Western ethnicity & 18 & $5 \cdot 2$ & & 16 & $3 \cdot 8$ & & \\
\hline Children of non-Western ethnicity & 193 & $55 \cdot 8$ & & 108 & $25 \cdot 4$ & & \\
\hline \multicolumn{8}{|l|}{ Educational level of the parents (\%) } \\
\hline Low & 110 & $36 \cdot 3$ & & 69 & $17 \cdot 1$ & & $<0.001$ \\
\hline Moderate & 108 & $35 \cdot 6$ & & 153 & $38 \cdot 0$ & & \\
\hline High & 85 & $28 \cdot 1$ & & 181 & $44 \cdot 9$ & & \\
\hline \multicolumn{8}{|l|}{ Number of siblings (\%) } \\
\hline 0 & 26 & $8 \cdot 4$ & & 31 & $7 \cdot 5$ & & $0 \cdot 117$ \\
\hline 1 & 129 & $41 \cdot 6$ & & 208 & $50 \cdot 5$ & & \\
\hline 2 & 80 & $25 \cdot 8$ & & 94 & $22 \cdot 8$ & & \\
\hline$\geq 3$ & 75 & $24 \cdot 2$ & & 79 & $19 \cdot 2$ & & \\
\hline
\end{tabular}

${ }^{*}$ As estimated by the $\chi^{2}$ test (independent categorical data). 
data: $\mathrm{OR}=1 \cdot 99,95 \%$ CI $1 \cdot 50,2 \cdot 65$; parent data: $\mathrm{OR}=2 \cdot 50$, $95 \%$ CI $1 \cdot 89,3 \cdot 31$ ), for those residing in the eastern region (child data: $\mathrm{OR}=2 \cdot 11,95 \%$ CI $1 \cdot 53,2 \cdot 92$ ), for boys (child data: $\mathrm{OR}=1 \cdot 42,95 \%$ CI 1.09, 1.87; parent data: $\mathrm{OR}=1 \cdot 76$, $95 \%$ CI $1 \cdot 34,2 \cdot 30$ ) and for parents of children of a nonWestern ethnicity (OR $=1 \cdot 40,95 \%$ CI $1 \cdot 01,1 \cdot 93)$.

At baseline, children of non-participating parents were more likely to be in the intervention group $(\mathrm{OR}=1 \cdot 79$, $95 \%$ CI $1 \cdot 10,2 \cdot 91)$, to live in the western region $(\mathrm{OR}=$ $2 \cdot 33,95 \% \mathrm{CI} 1 \cdot 34,4 \cdot 07)$, to be a boy $(\mathrm{OR}=1 \cdot 56,95 \% \mathrm{CI}$ $1 \cdot 01,2 \cdot 42)$ and have a non-Western ethnicity $(\mathrm{OR}=3 \cdot 09$, $95 \%$ CI $1 \cdot 82,5 \cdot 22$ ). At second follow-up, the difference regarding region of residence was no longer significant while the differences in gender $(\mathrm{OR}=1 \cdot 84,95 \% \mathrm{CI} 1 \cdot 40$, $2 \cdot 43)$, study group $(\mathrm{OR}=4 \cdot 61,95 \% \mathrm{CI} 3 \cdot 45,6 \cdot 17)$ and ethnicity $(\mathrm{OR}=1 \cdot 40,95 \% \mathrm{CI} 1 \cdot 01,1 \cdot 94)$ remained.

\section{Characteristics of the participants}

As shown in Table 1, the study sample consisted of slightly more girls than boys, in the intervention as well as in the control group. The majority of the children were of native Dutch origin, in particular in the control group. At baseline, the age of all children ranged between 8.5 and $11 \cdot 8$ years; for parents this was $25 \cdot 2-61 \cdot 0$ years.

\section{Fruit and vegetable intakes (primary outcomes)}

Table 2 shows the observed mean values for the childreported intakes of $\mathrm{F} \& \mathrm{~V}$, as well as the mean parentreported intakes of $\mathrm{F} \& \mathrm{~V}$, for the baseline measurement and for the measurement at second follow-up.

At second follow-up the unadjusted analyses showed higher child-reported fruit intake in the intervention than in the control group (Table 2), which remained significant after adjustment for potential confounders (difference, pieces/d: 0.145, 95\% CI 0.004, 0·286; Table 3). Results of the parent-reported data support these observations (adjusted difference, pieces/d: 0.185, 95\% CI 0.030, 0.340; Tables 2 and 3).

A significant interaction $(P=0.013)$ with parental educational level was found for child-reported fruit intake, but after stratification no significant effect sizes were found in either group most likely due to lack of power (data not shown). This was also observed in the parent-reported data (data not shown).

At second follow-up the children in the intervention group reported a significantly higher unadjusted vegetable intake than the children in the control group ( $P=0 \cdot 025$; Table 2$)$, but after adjustment for potential confounders this difference was no longer significant (Table 3). Also for the parent-reported vegetable intake, no significant difference was observed after adjustment for potential confounders (Table 3).

\section{Determinants of fruit intake (secondary outcomes)}

All analyses on determinants of fruit intake were not adjusted for Western ethnicity because of small samples

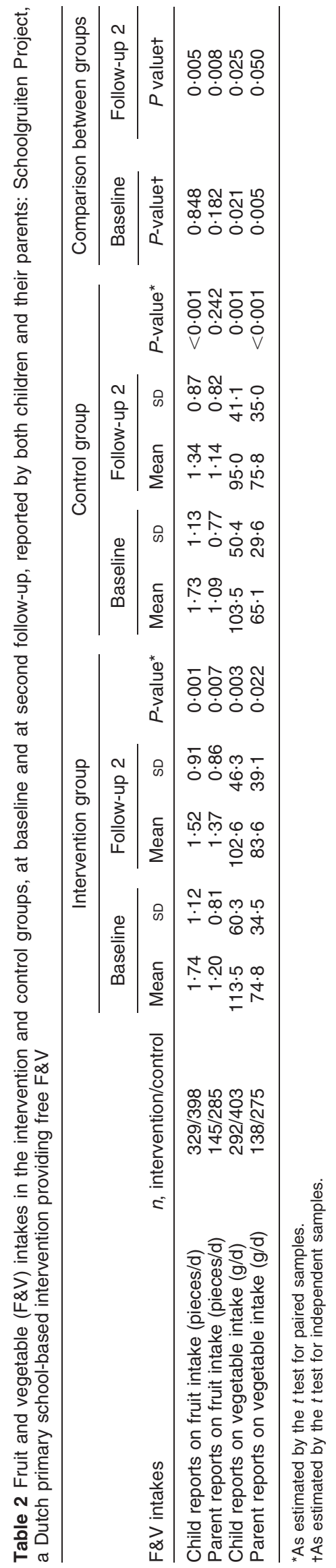


Table 3 Indicators of the effect of the intervention regarding fruit and vegetable (F\&V) intake from multilevel regression analyses conducted on both child reports and parent reports: Schoolgruiten Project, a Dutch primary school-based intervention providing free F\&V

\begin{tabular}{lccc}
\hline Primary outcomes & $n$ & $\beta \dagger$ & $95 \% \mathrm{Cl}$ \\
\hline Child reports on fruit intake (pieces/d) & 667 & $0 \cdot 145$ & $0.004,0 \cdot 286$ \\
Parent reports on fruit intake (pieces/d) & 423 & $0 \cdot 185$ & $0.030,0.340$ \\
Child reports on vegetable intake (g/d) & 635 & 0.67 & $-7 \cdot 00,8 \cdot 34$ \\
Parent reports on vegetable intake (g/d) & 407 & 0.56 & $-7 \cdot 72,8 \cdot 85$ \\
\hline
\end{tabular}

*Analyses are adjusted for children's age, gender, ethnicity, education level of the parents, region of residence of the children, and baseline levels of fruit or vegetable consumption.

$+\beta$ indicates difference in primary outcome in the intervention group compared with the control group.

and empty cells; therefore this group was merged with the native Dutch group.

According the child-reported data, the unadjusted results indicated that children in the intervention group were more likely to know the recommendations for fruit intake, were more often allowed to take fruit without asking and also perceived higher fruit availability at home at second follow-up (Table 4).

After adjustments and stratification (because of a significant interaction with gender, $P=0 \cdot 021$ ) a significant intervention effect was still observed among boys for knowledge of the recommendations of fruit intake. Furthermore, adjusted analyses showed that children in the intervention group in the eastern region were more likely to be in the stable low group for their preferences for fruit between baseline and second follow-up.

At baseline, $79 \%$ of the parents of all children reported that their child liked fruit or liked fruit very much. This proportion did not differ between the intervention and control group at baseline or at second follow-up (Table 4). Although the children in the intervention group seemed to have higher taste preferences at follow-up (unadjusted analyses, Table 4), adjusted analyses showed that this difference was not significant (Table 5). Finally, unadjusted analyses showed that accessibility had increased in both the intervention group and the control group (Table 4), which resulted in no significant intervention effect (Table 5).

\section{Mediation analyses}

Following the steps of mediation analyses, first we explored if (i) the intervention was independently associated with the appreciation of the project (path A, Fig. 1). Table 6 shows that the children in the intervention group (89.7\%) appreciated the project more than the children in the control group $(62.5 \%)$. Tested in a multinomial regression model, this association appeared significant; the children of the intervention group were more likely to positively appreciate the intervention.

Second, we explored if (ii) the appreciation of the project was independently associated with F\&V intake (path B). In a regression model with two dummies for appreciation of the project, this association also appeared significant; the children who appreciated the project positively increased their fruit intake. Children's appreciation of the project was therefore further explored as a possible mediator.

The last two steps explored (iii) if the intervention was associated with F\&V intake (path C) and (iv) if the association between intervention and F\&V intake decreased substantially when the analysis was adjusted for appreciation of the project. In the regression model to test these steps, this association appeared also significant. Table 7 shows the change in the regression coefficient for fruit intake when adjustments were made for appreciation of the project (step iii). Adding children's appreciation of the project to the model, the regression coefficient decreased from $0 \cdot 12$ to $0 \cdot 04(66 \cdot 4 \%$ decrease; step iv).

\section{Discussion}

The present study indicates that the Schoolgruiten Project had a small but significant positive effect on children's fruit intake, supported by child- and parent-reported data. This is in accordance with the findings of other intervention studies, such as the European Pro Children Study and the Norwegian Fruit and Vegetables Makes the Marks (FVMM) project, which observed somewhat higher effect sizes $^{(20,21)}$ at longer-term follow-up.

Unfortunately, in the current study, the regression coefficients for vegetable intake were very small and not significant. This may be due to the difficulty of increasing vegetable intake levels in the school environment in The Netherlands. Within Dutch eating habits, vegetables are eaten at the main (evening) meal, i.e. in the home environment. No school meals are offered in Dutch primary schools, which makes promotion of vegetable consumption during school hours more difficult. In a country like Sweden, where school meals are offered and have to comply to dietary recommendations, a higher consumption of vegetables at school is possible ${ }^{(22)}$. To increase vegetable consumption among Dutch children involvement of the parents is of key importance; for example, with newsletters and homework assignments for both parents and their children to perform together ${ }^{(2)}$.

Contrary to the Pro Children Study and FVMM project, the Schoolgruiten Project was initiated outside an academic centre; it was planned, developed and implemented by a 


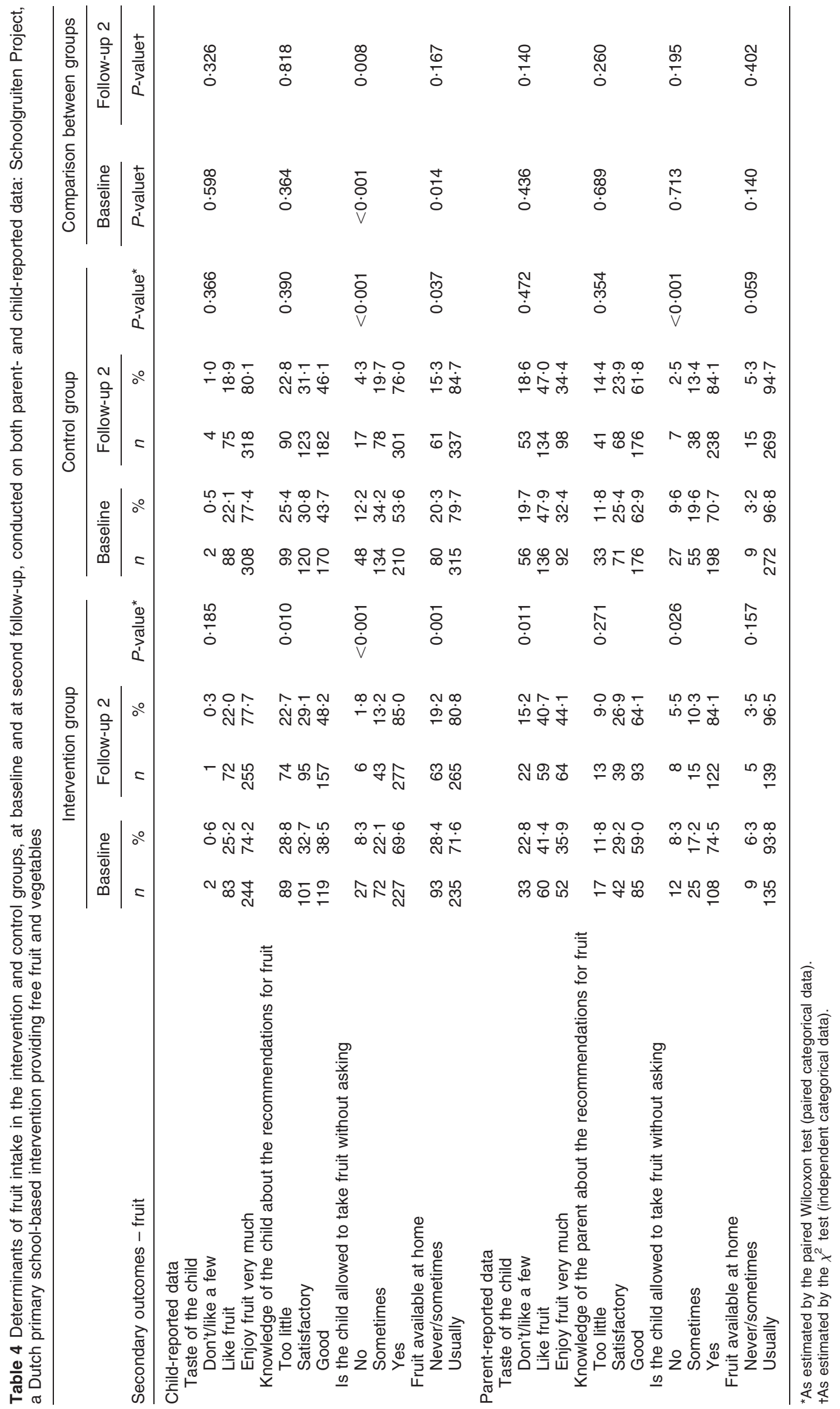




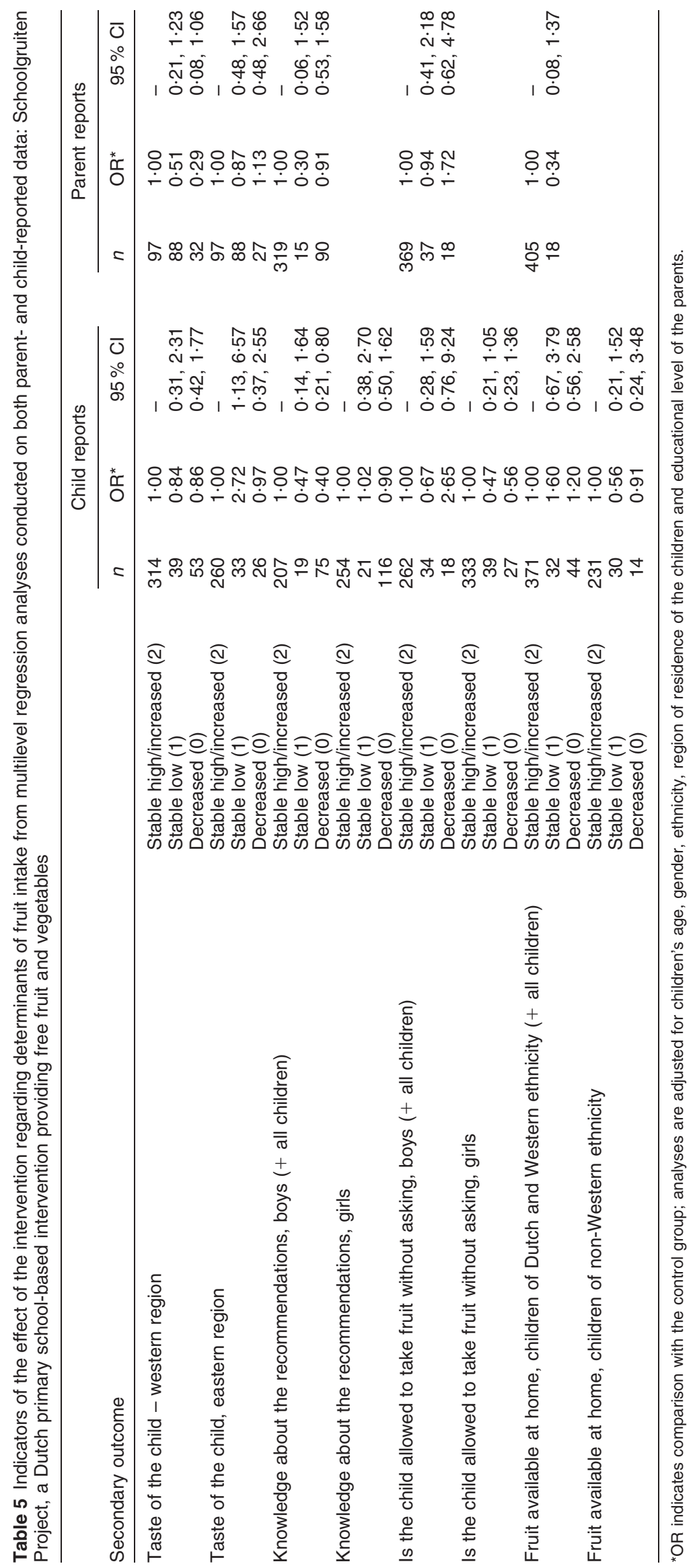


Table 6 Appreciation of the project or a fruit and vegetable (F\&V)-promoting project in general for both the intervention and control groups, at baseline and at second follow-up: Schoolgruiten Project, a Dutch primary school-based intervention providing free $\mathrm{F} \& \mathrm{~V}$

\begin{tabular}{|c|c|c|c|c|}
\hline \multirow{2}{*}{$\begin{array}{l}\text { Appreciation of the project or an } \\
\text { F\&V-promoting project in general }\end{array}$} & \multicolumn{2}{|c|}{ Intervention group } & \multicolumn{2}{|c|}{ Control group } \\
\hline & $n$ & $\%$ & $n$ & $\%$ \\
\hline \multicolumn{5}{|l|}{ Baseline measurement } \\
\hline Negative & 25 & $8 \cdot 1$ & 91 & $23 \cdot 4$ \\
\hline Neutral & 22 & $7 \cdot 1$ & 52 & $13 \cdot 4$ \\
\hline Positive & 261 & $84 \cdot 7$ & 246 & $63 \cdot 2$ \\
\hline \multicolumn{5}{|l|}{ Second follow-up } \\
\hline Negative & 17 & $5 \cdot 6$ & 74 & $19 \cdot 3$ \\
\hline Neutral & 14 & $4 \cdot 6$ & 70 & $18 \cdot 2$ \\
\hline Positive & 271 & $89 \cdot 7$ & 240 & $62 \cdot 5$ \\
\hline
\end{tabular}

Table 7 Effect of adjustment for appreciation of the project or a fruit and vegetable (F\&V)-promoting project in general, as mediator in the association between intervention and fruit intake at second follow-up: Schoolgruiten Project, a Dutch primary school-based intervention providing free F\&V

\begin{tabular}{lccrr}
\hline & $n$ & Regression coefficient & \multicolumn{1}{c}{$95 \% \mathrm{Cl}$} & $\%$ \\
\hline Unadjusted effect-size of fruit intake & 612 & $0 \cdot 144$ & $0 \cdot 00,0 \cdot 29$ & \\
Adjusted effect-size of fruit intake & 612 & $0 \cdot 116$ & $-0 \cdot 03,0 \cdot 27$ & $-19 \cdot 4$ \\
+ Appreciation of the project at second follow-up & 612 & 0.039 & $-0 \cdot 11,0 \cdot 19$ & $-66 \cdot 4$ \\
\hline
\end{tabular}

public-private partnership of the Netherlands Nutrition Centre Foundation with the promotion office of the Dutch F\&V producers. This partnership did try to combine intervention strategies that were tailored to important mediators of F\&V intake in primary-school children, but, because of time constraints, was not able to work carefully according to established planning models for health promotion such as the Intervention Mapping protocol, as was done for the Pro Children intervention ${ }^{(23)}$, for example.

The Schoolgruiten intervention aimed at different determinants of $\mathrm{F} \& \mathrm{~V}$ : increasing taste preference, availability and accessibility of F\&V through the free delivery of $\mathrm{F} \& \mathrm{~V}$ at school and improving knowledge about the recommended intake levels of $\mathrm{F} \& \mathrm{~V}$ through the school curriculum. We found that knowledge of recommended intake levels was improved among boys in the intervention group at second follow-up, probably as a result of using the curriculum materials. Unfortunately, we found no effects on perceived accessibility or availability, while we found some effects on perceived accessibility at first (short-term effects) follow-up ${ }^{(1)}$. Furthermore, no effects were observed on potential behavioural determinants in the parent-reported data.

Mediation analyses supported previous findings that children's appreciation of the project was associated with the intervention effects on fruit intake. This result suggests that children should be consulted in the development of school-based interventions so that appreciation of the programme is promoted, in order to optimize intervention effects.

Evaluation of school-based healthful nutrition promotion interventions should be based on accurate and valid assessments of intake levels and mediators of intakes ${ }^{(24)}$.
Collecting accurate intake data based on observations or biomarkers is often possible in smaller-scale, carefully controlled efficacy studies, but not in larger-scale studies in real-life settings. Collecting blood samples in children introduces bias because of low participation rates ${ }^{(25,26)}$. FFQ are therefore generally used. However, these questionnaires rely on participants' memory and cognition, which may influence the accuracy of the reported intake; but this bias is believed to be the same in the control as in the intervention group. In the present study we used both child- and parent-reported intake levels for assessment of effects. The regression coefficients for fruit intake were approximately the same in both the child-reported data and the parent-reported data, which makes the evidence for an intervention effect stronger. Also, the regression coefficients for vegetable intake were approximately the same for parents' reports and children's reports.

In another publication concerning the same study population, Tak et al. concluded that the level of agreement between child-reported F\&V intakes and parent-reported $\mathrm{F} \& \mathrm{~V}$ intakes was low at the baseline measurement ${ }^{(12)}$. In the present study the reported intakes at second follow-up (2 years later) were much more alike, although the children still reported somewhat higher intake levels. This result further supports the earlier conclusion that child and parent reports tend to be more similar for somewhat older schoolchildren.

A disadvantage of the research design applied in the present study is that randomisation was not possible, since the Dutch government had indicated the intervention cities. Our analyses showed some baseline differences between intervention and control groups; the fact that schools were not randomly allocated may have introduced bias. Notably, 
the baseline difference for the parent-reported vegetable intake was significant. An explanation could be that there were relatively more children with a non-Western ethnicity in the intervention group compared with the control group. Tak et $a l^{(1)}$ concluded elsewhere about this project that children with non-Western ethnicity reported higher vegetable consumption than children with Dutch ethnicity. Therefore, we adjusted all analyses for baseline differences to solve this problem. However, it may still have affected the results of the intervention since it was more difficult to further increase the vegetable intake among the children of the intervention group compared with the children from the control group.

Another bias may have occurred due to some selective dropout; however, loss to follow-up was not a consequence of an autonomous decision of the child, but was primarily caused by dropout of eight schools and in some cases based on parental decisions.

\section{Conclusion}

The present study provides some evidence that the Schoolgruiten intervention was effective in increasing children's fruit intake and increasing the knowledge of recommended intake levels among boys at 2-year follow-up, and confirms results from earlier studies indicating that school-based F\&V schemes with additional school curriculum activities can have significant effects. Furthermore, children's appreciation of the project appeared to mediate the intervention effects for fruit intake and future interventions should take this into account in order to achieve positive intervention effects.

\section{Acknowledgements}

Sources offunding: Funding for the Schoolgruiten study was provided by the Dutch Ministry for Health, Welfare and Sport and Holland Produce Promotion in Zoetermeer. The contribution of N.I.T. was funded by the World Cancer Research Fund (grant number: 2004/05). The contribution of S.J.t.V. was funded by the Netherlands Organization for Health Research and Development (ZonMw) Program for Prevention (no. 6200•0003).

Conflict of interest declaration: The authors declare that they have no competing interests.

Authorship responsibilities: N.I.T. collected and analysed the data and drafted the manuscript. S.J.t.V. and J.B. participated in the study design and provided critical revision of the manuscript. All three authors have read and approved the final manuscript.

Acknowledgements: We thank the schools and schoolchildren for participating in this study.

\section{References}

1. Tak NI, te Velde SJ \& Brug J (2007) Ethnic differences in 1-year follow-up effect of the Dutch Schoolgruiten
Project - promoting fruit and vegetable consumption among primary-school children. Public Health Nutr 10, 1497-1507.

2. Wind M (2007) Pro Children; The Development, Implementation and Evaluation of a School-based Intervention to Promote Fruit and Vegetable Intake among 10-13 yearold European Schoolchildren. Rotterdam: Erasmus Medical Center.

3. Bere E \& Klepp KI (2004) Reliability of parental and selfreported determinants of fruit and vegetable intake among 6th graders. Public Health Nutr 7, 353-356.

4. Wind M, De Bourdeaudhuij I, te Velde SJ, Sandvik C, Klepp KI, Due P \& Brug J (2006) Correlates of fruit and vegetable consumption among 11-year-old BelgianFlemish and Dutch schoolchildren. J Nutr Educ Behav 38, 211-221.

5. De Bourdeaudhuij I, Klepp KI, Due P, Rodrigo CP, De Almeida MDV, Wind M, Krolner R, Sandvik C \& Brug J (2005) Reliability and validity of a questionnaire to measure personal, social and environmental correlates of fruit and vegetable intake in 10-11-year-old children in five European countries. Public Health Nutr 8, 189-200.

6. Blanchette L \& Brug J (2005) Determinants of fruit and vegetable consumption among 6-12-year-old children and effective interventions to increase consumption. J Hum Nutr Diet 18, 431-443.

7. Baranowski T, Cullen KW \& Baranowski J (1999) Psychosocial correlates of dietary intake: advancing dietary intervention. Annu Rev Nutr 19, 17-40.

8. Wardle J, Herrera ML, Cooke L \& Gibson EL (2003) Modifying children's food preferences: the effects of exposure and reward on acceptance of an unfamiliar vegetable. Eur J Clin Nutr 57, 341-348.

9. Haraldsdottir J, Thorsdottir I, de Almeida MD, Maes L, Perez Rodrigo C, Elmadfa I \& Frost Andersen L (2005) Validity and reproducibility of a precoded questionnaire to assess fruit and vegetable intake in European 11to 12-year-old schoolchildren. Ann Nutr Metab 49, 221-227.

10. Kristjansdottir AG, Andersen LF, Haraldsdottir J, De Almeida MDV \& Thorsdottir I (2006) Validity of a questionnaire to assess fruit and vegetable intake in adults. Eur J Clin Nutr 60, 408-415.

11. Statistics Netherlands (2005) Website of Statistics Netherlands. http://www.cbs.nl/nl-NL/menu/methoden/begrippen/alfabet/ n/niet-westerse-allochtoon.htm (accessed December 2005).

12. Tak NI, te Velde SJ, De Vries JHM \& Brug J (2006) Parent and child reports of fruit and vegetable intakes and related family environmental factors show low levels of agreement. J Hum Nutr Diet 19, 275-285.

13. Reinaerts E, de Nooijer J \& de Vries NK (2007) Parental versus child reporting of fruit and vegetable consumption. Int J Behav Nutr Phys Act 4, 33.

14. Twisk JWR \& Proper K (2004) Evaluation of the results of a randomized controlled trial: how to define changes between baseline and follow-up. J Clin Epidemiol 57, 223-228.

15. Baron RM \& Kenny DA (1986) The moderator-mediator variable distinction in social psychological research: conceptual, strategic, and statistical considerations. J Pers Soc Psychol 51, 1173-1182.

16. MacKinnon DP (1994) Analysis of mediating variables in prevention and intervention research. In: Scientific Methods for Prevention Intervention Research. NIDA Research Monograph no. 139, DHHS Publication no. 94-3631, pp. 127-153 [A Cazares \& LA Beatty, editors]. Washington, DC: US Department of Health and Human Services.

17. MacKinnon DP, Krull JL \& Lockwood CM (2000) Equivalence of the mediation, confounding and suppression effect. Prev Sci 1, 173-181. 
18. MacKinnon DP, Lockwood CM, Hoffman JM, West SG \& Sheets V (2002) A comparison of methods to test mediation and other intervening variable effects. Psychol Methods $\mathbf{7}$, 83-104.

19. Rasbash J, Steele F, Browne W \& Prosser B (2004) A User's Guide to MLwiN version 2.02. London: Institute of Education.

20. te Velde SJ, Brug J, Wind M, Hildonen C, Bjelland M, PerezRodrigo C \& Klepp KI (2008) Effects of a comprehensive fruit- and vegetable-promoting school-based intervention in three European countries: the Pro Children Study. Br J Nutr 99, 893-903.

21. Bere E, Veierod M, Skare O \& Klepp KI (2007) Free school fruit - sustained effect three years later. Int J Behav Nutr Phys Act 4, 5.

22. Yngve A, Wolf A, Poortvliet E et al. (2005) Fruit and vegetable intake in a sample of 11-year-old children in 9
European countries: the Pro Children cross-sectional survey. Ann Nutr Metab 49, 236-245.

23. Pérez-Rodrigo $C$, Wind $M$, Hildonen $C$, Bjelland $M$, Aranceta J, Klepp KI \& Brug J (2005) The Pro Children intervention: applying the Intervention Mapping protocol to develop a school-based fruit and vegetable promotion programme. Ann Nutr Metab 49, 267-277.

24. Klepp KI, Pérez-Rodrigo C, De Bourdeaudhuij I et al. (2005) Promoting fruit and vegetable consumption among European schoolchildren: rationale, conceptualization and design of the Pro Children Project. Ann Nutr Metab 49, 212-220.

25. Livingstone MBE \& Robson PJ (2000) Measurement of dietary intake in children. Proc Nutr Soc 59, 279-293.

26. Hammond J, Nelson M, Chinn S \& Rona RJ (1993) Validation of a food frequency questionnaire for assessing dietary intake in a study of coronary heart disease risk factors in children. Eur J Clin Nutr 47, 242-250. 